\title{
Unsteady flow field around a human hand and propulsive force in swimming
}

\author{
K. Matsuuchi ${ }^{\mathrm{a}, *}$, T. Miwa ${ }^{\mathrm{b}}$, T. Nomura ${ }^{\mathrm{b}}$, J. Sakakibara ${ }^{\mathrm{a}}$, \\ H. Shintani ${ }^{\text {a }}$, B.E. Ungerechts ${ }^{\mathrm{c}}$ \\ ${ }^{a}$ Graduate School of Systems and Information Engineering, University of Tsukuba, \\ Tsukuba 305-8573, Japan \\ ${ }^{\mathrm{b}}$ Graduate School of Comprehensive Human Sciences, University of Tsukuba, \\ Tsukuba 305-8573, Japan \\ ${ }^{\mathrm{c}}$ Institute of Sport Sciences, University of Bielefeld, D-33615 Bielefeld, Germany
}

\begin{abstract}
Much effort has been undertaken for the estimation of propulsive force of swimmers in the front crawl. Estimation is typically based on steady flow theory: so-called the quasi-steady analysis. Flow fields around a swimmer, however, are extremely unsteady because the change direction of hand produces unsteady vortex motions. To evaluate the force correctly, it is necessary to know the unsteady properties determined from the vortex dynamics because that unsteadiness is known to make the force greater. Unsteady flow measurements were made for this study using a sophisticated technique called particle image velocimetry (PIV) in several horizontal planes for subjects swimming in a flume. Using that method, a hundred time-sequential flow fields are obtainable simultaneously. Each flow field was calculated from two particle images using the cross-correlation method. The intensity of vortices and their locations were identified. A strong vortex was generated near the hand and then shed by directional change of the hand in the transition phase from in-sweep to out-sweep. When the vortex was shed, a new vortex rotating in the opposite direction around the hand was created. The pair of vortices induced the velocity component in the direction opposite to the swimming. Results of this study show that the momentum change attributable to the increase of this velocity component is the origin of thrust force by the hand.
\end{abstract}

Key words: propulsive force, front crawl, PIV, unsteady flow PACS: 47.15.ki, 47.80.Jk

\footnotetext{
* Corresponding author.

Email address: matsuuch@kz.tsukuba.ac.jp (K. Matsuuchi).
}

Preprint submitted to Journal of Biomechanics 6 October 2008 


\section{Introduction}

The first important contribution related to the mechanism of propulsion in a swimming stroke was made by Counsilman (1971). That mechanism has been discussed by dividing the force into two components: a lift component normal to the hand motion and a drag one parallel to it. He pointed out the importance of lift force rather than drag force. The next critical contribution was made by Schleihauf (1979). He measured the lift and drag forces on hand models various geometrical configurations of flow. Berger et al. (1995) carried out similar measurements using models consisting of hand and arm motions for a fixed geometrical configuration in flow and obtained the result consistent with that by Schleihauf. Subsequently, Bixler and Riewald (2002) used a numerical approach in a similar situation to that of the experiments described above.

The approach described above is called the quasi-steady analysis, which depends on the assumption that the flow at each instance is nearly steady. This quasi-steady approach is simple in application, but its accuracy is questionable. Toussaint et al. (2002) pointed out that the calculated propulsive forces based on data obtained by Berger et al. (1999) were 17\% lower than the measured active drag. Moreover, Sanders (1999) reported much lower coefficients (about $50 \%$ ) of lift and drag. The difference between the two seems to result from the inclusion of wave drag in the former. Even for the higher estimation, the quasi-steady analysis underestimates the coefficients. These discrepancies arise from the simplistic assumption that the flow has no temporal changes. Unsteady effects occured by the change actions of hand, such as the directional changes of hand, are ignored in the approach. This characteristic must be considered when investigating the basic properties of force.

Maximum unsteady lift is often large compared with the steady lift. The lift acting in such unsteady situations is designated as the "dynamic lift". According to Izumi and Kuwahara (1983), the maximum lift coefficient becomes twice as large as the steady one for the case of an impulsively starting ellipsoid. Several researchers have noted the importance of unsteadiness in swimming strokes arising from vortex movement. For instance, Ungerechts (1986) pointed out the importance of the turning phase of the leg kick in the breaststroke and found that the acceleration peak of the body agrees approximately with the turning phase of the feet. Arellano (1999) investigated the vortices generated in underwater swimming, and suggested that the size and movement of the vortex seem to be related to propulsion obtained through hand and foot movements. In addition, Arellano et al. (2002) described the difference between efficient and less efficient swimmers from the perspective of gener-

ated patterns of vortices. In relation to these observations, there remain some doubts related to the time delay between the force peak and vortex generation. 
The delay is closely related to the fundamental assumption of the quasi-steady state model, and has been challenged on basic theoretical grounds by invoking the mathematical work of Wagner (1925), who estimated that maximal lift is produced only after several chord lengths of travel (Ellington (1984)). However, according to Izumi and Kuwahara (1983) and Dickinson and Götz (1993) the maximal lift was attained after only a few chord lengths' travel. Considering these results, the findings in swimming seem to be sufficiently explanatory.

This study is intended to elucidate the generation mechanism of propulsive force by hand actions and thereby expand the knowledge of unsteady mechanisms that might be used by real swimmers. We first consider the simplest model of the generation of a vortex called the "starting vortex". This example is depicted in Fig. 1, where a hydrofoil is at rest initially and then moves from left to right. A vortex remains at the place where the hydrofoil was located initially. This is called the starting vortex. In the example, the vortex rotates clockwise, but a bound vortex around the hydrofoil, which rotates counterclockwise, is generated.

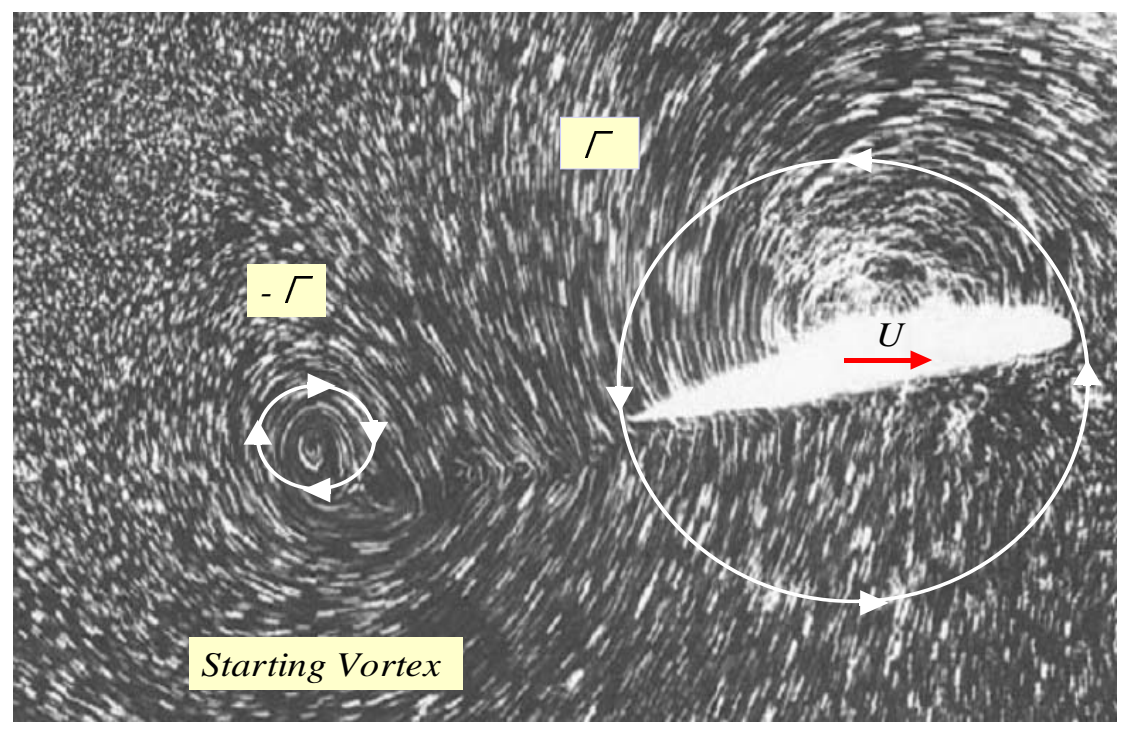

Fig. 1. Starting vortex and bound vortex around a hydrofoil. The starting vortex with circulation $-\Gamma$ remains where the hydrofoil started. A new fluid circulation $\Gamma$ is produced around the hydrofoil.

A pair of vortices can generate momentum downward, as illustrated schematically in Fig. 2. One clockwise-rotating vortex is shed from a hydrofoil and fixed in space; the other, which is located around the hydrofoil, moves to the right at constant speed relative to the clockwise-rotating vortex. Denoting the momentum of water at $t$ as $P(t)$, the momentum at $t+\Delta t$ is increased to $P(t+\Delta t)=P(t)+\Delta P$ because the counterclockwise vortex around the hy- 
drofoil generates new downward momentum by moving to the right. We have, from Newton's second law, for the force $L$ on the hydrofoil,

$$
L=-\frac{P(t+\Delta t)-P(t)}{\Delta t}=\frac{\Delta P}{\Delta t}
$$

The minus sign of the right-hand side denotes that the direction of the lift is in the opposite direction of the momentum. It is natural to postulate that the momentum made by the movement of a hydrofoil is proportional to the fluid density $\rho$, the magnitude of the vortex, $\Gamma$, and the distance between two vertices, $s$. Consequently, the momentum $P$ is written as $-\rho \Gamma s$ which is called the impulse, or virtual momentum (see Lamb, 1932; the minus sign indicates a downward direction). Therefore, we have the well-known Kutta-Joukowski theorem $L=\rho U \Gamma$ because $U=\mathrm{d} s / \mathrm{d} t$.

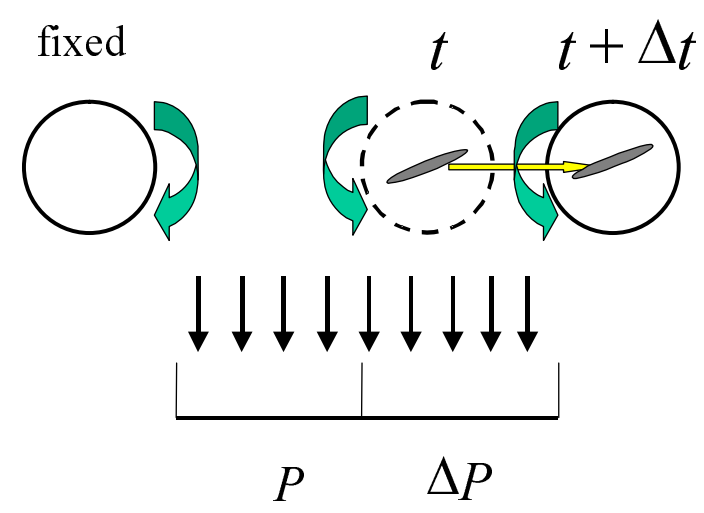

Fig. 2. Momentum generation by a pair of counter-rotating vortices. The vortex with clockwise rotation is fixed in space, but that with counterclockwise rotation is moving to the right.

Swimmers can generate unsteady forces in addition to the steady forces, through the processes of vortex production and shedding. For purposes of illustration of the generation the hand movement will be considered only in a horizontal plane. The motion of a hand moving is portrayed in Fig. 3. Instead of fingers, an ellipsoid is adopted as the position of a hand. Swimmers change the direction of motion, e.g., from in-sweep to out-sweep. In the context of the force generation mechanism of insect and bird flights, the force is discussed in relation to lift force. Here we discuss the generation of lift force. Presume that a swimmer moves from right to left at constant velocity $\boldsymbol{u}_{b}$. The trace of a hand and the hand positions at $t=t_{1}$ and at a later time $t_{2}$ are depicted in the figure. A trace is drawn relative to the body and the relative hand velocity is denoted as $\boldsymbol{u}_{h}$. The resultant hand velocity relative to the flow is given by the vector sum of the two vectors $\boldsymbol{u}_{b}$ and $\boldsymbol{u}_{h}$. Therefore, the flow velocity relative to the hand is given as $-\left(\boldsymbol{u}_{b}+\boldsymbol{u}_{h}\right)$. Figure 3 shows the vector triangles of the 


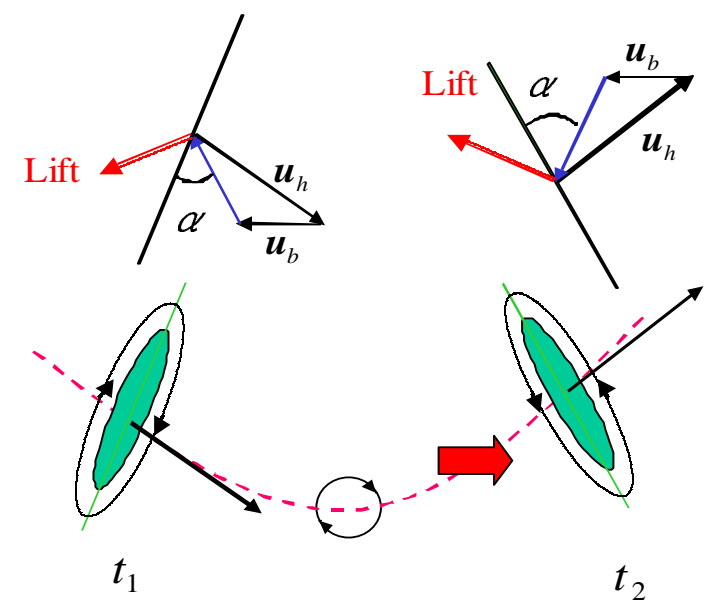

Fig. 3. Simplified 2D explanation of the generation of momentum. Circulation around a hand changes its sign in the transition phase. The thick arrow denotes the momentum induced using a pair of vortices: a bound vortex and a free one.

three vectors in the upper part of the figure. The flow relative to the hand has an angle of attack of $\alpha$. The lift force is generated in the direction perpendicular to the flow, as depicted in Fig. 3. Noting that a bound vortex rotates clockwise at time $t_{1}$, the lift is found to be in the direction depicted in the figure. At $t_{2}$ after the in-sweep, the rotation must be changed from clockwise to counterclockwise because the direction of the velocity relative to the hand is changed. Fluid mechanics dictate that the total intensity of vortices cannot be changed. Consequently, the bound vortex, which was rotating clockwise around the hand at $t=t_{1}$ must be shed to free water; a new bound vortex, rotating counterclockwise, is produced instead. The shed and bound vortices constitute a pair of counter-rotating vortices like a starting vortex and bound one. A vortex pair generates momentum. Lift is thereby exerted on the object as the reaction of momentum change. We refer to this phenomenon as the "change circulation".

\section{Methods}

We performed experiments using a flume installed at the University of Tsukuba (Igarashi Industrial Works Co. Ltd.). The test section is $4.6 \mathrm{~m}$ long, $2 \mathrm{~m}$ wide, and $1.5 \mathrm{~m}$ high $(1.2 \mathrm{~m}$ water depth); it can provide a maximum flow of 2.5 $\mathrm{m} / \mathrm{s}$. The flow speed was set at about $1.2 \mathrm{~m} / \mathrm{s}$ in most cases.

We used a recently developed technique called particle image velocimetry 

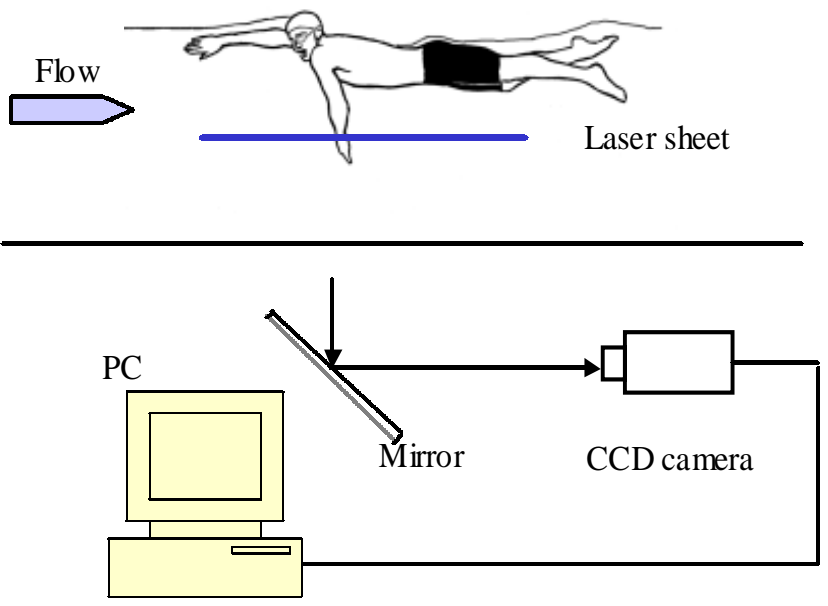

Fig. 4. PIV system and experimental setup.

(PIV). The laser light must be intense to adapt PIV technique to a wide region around the human hand. A Nd-YAG laser (Solo PIV 120; New Wave Research Inc.) was chosen for this purpose. As tracers, 50- $\mu$ m-diameter nylon particles were used. The horizontal level of the laser sheet was set at the depth of about $55 \mathrm{~cm}$ below the water surface, depending on the subject. The optimum level is adjusted so that the hand cuts the illuminated plane when it is just in the phase from in-sweep to out-sweep. Illuminated particle images were reflected by a mirror and then captured by a CCD camera (ES1.0; Eastman Kodak Co.). The mirror and the camera were set on the same lower floor, located $95 \mathrm{~cm}$ below the bottom of the flume. Finally, those were stored in the host memory of a PC via image grabber (Coreco Imaging Inc.). Our data acquisition system is presented in Fig. 4. The cross-correlation method was used as the algorithm for determining the flow field (see Sakakibara et al. $(2004))$.

The particle displacement that occurs after a short period of time $\Delta t$ determines the velocity as

$$
u=\frac{\Delta x}{\Delta t}, \quad v=\frac{\Delta y}{\Delta t}
$$

In the present experiment, we set $\Delta t=1 \mathrm{~ms}$.

Our PIV system can obtain 100 pairs of images at once for velocity fields. The sampling period of the velocity fields was chosen as $70 \mathrm{~ms}$. Therefore, 14 planes per second were detected. Our approach is limited to a two-dimensional (2D) analysis in horizontal planes. 
Next, we describe an important quantity called the vorticity for considering the force generation attributable to the vortex generation and shedding. The vorticity is a measure of the magnitude of rotation of a small element of fluid, or that of a vortex. The component normal to the measured plane, $x-y$ plane, is defined as

$$
\zeta=\frac{\partial u}{\partial y}-\frac{\partial v}{\partial x}
$$

where $(u, v)$ respectively signify the velocity components in the $x$ - and $y-$ directions. For example, the element rotating with the angular velocity $\Omega$ rotating about the $z$-axis gives $\zeta=2 \Omega$ (Tritton (1988), Lugt (1995)).

Five swimmers volunteered for this study. Most of the subjects were members of the Tsukuba University Swim Team.

\section{Results}

We selected subject A and subject B as typical examples among the subjects. First we present an example for subject A, a trained male swimmer. A CCD camera set under a flume picks up a mirror image of the trace of a hand path in the plane (see Fig. 4). Figure 5(a) depicts the particle image and the swimmer including a hand; Fig. 5(b) portrays the velocity vector and vorticity defined in Eq.(3) in the same plane inside the white rectangle of (a). The flume velocity is directed from left to right and is $1.2 \mathrm{~m} / \mathrm{s}$ in magnitude. The image is produced just when the hand is at the transition phase from in-sweep to out-sweep. The hand and body outlines were superimposed in (a) to clarify the hand position relative to the body. In (b), the mean $x$-component had already been subtracted, so that the induced velocity is clarified. The color is used as an index of the magnitude of vortices or the vorticity measured in $1 / \mathrm{s}$, as denoted in the color bar and also the direction of rotation. The blue color corresponds to the clockwise vortex and the red to the counterclockwise one. A strong counterclockwise vortex is generated above the dorsal side (the left side of the fingers) near the little and third fingers. The cross mark denotes the position of the maximum vorticity. The clockwise vortices colored blue were shed as a free vortex, which had been a bound vortex around a hand during the in-sweep motion. The open circle is the position of the minimum vorticity. Another vortex of large scale is also visible, rotating clockwise near the vortex of the minimum vorticity. These consist of a pair of vortices.

Next, we present a series of velocity and vorticity fields in Fig. 6 for the same subject as that shown in Fig. 5. The real time $t$ is given as $t=0.07 \times(k-1)$ $\mathrm{s}$, where $k$ is an integer. The initial instant for $k=0$ is chosen arbitrarily. 


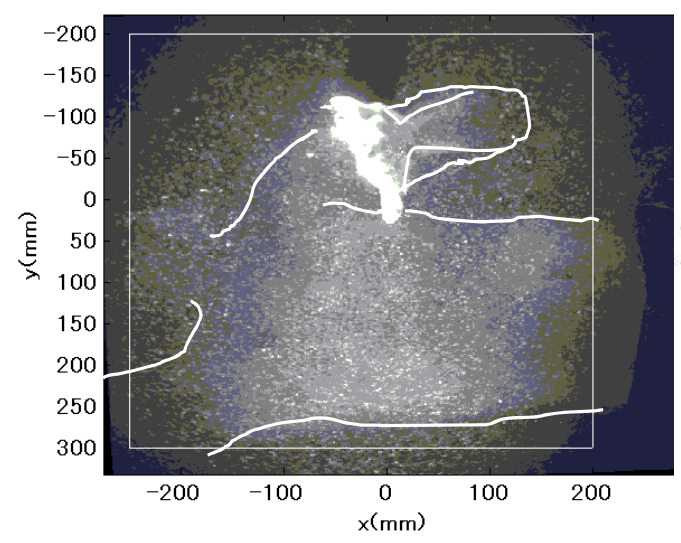

(a)

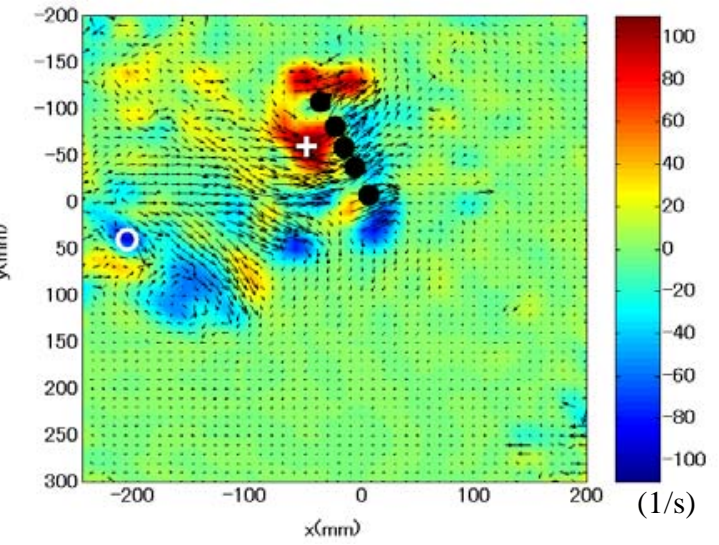

(b)

Fig. 5. An example of the image and velocity field in the horizontal plane. The flume speed was set at $1.2 \mathrm{~m} / \mathrm{s}$. Panel (a) shows a mirror image of the trunk and the right hand. The five filled circles in (b) correspond to the fingers. The color in (b) represents the strength of the vorticity, measured in units of $1 / \mathrm{s}$. The minimum value of the vorticity is $-99.7 \mathrm{~s}^{-1}$ at the open circle and the maximum one $151.1 \mathrm{~s}^{-1}$ at the cross mark.

The first figure corresponds to the 42nd stage $(k=42)$, in which the hand is in an in-sweep position. The fingers are simply replaced by an ellipsoid. The next stage is the instant when a transition from in-sweep to out-sweep has just occurred. The strong counterclockwise vortex is visible at the dorsal side near the third finger. The cross mark stands for the position where the magnitude is maximum. A deep blue area is found at the upstream side of the thumb. Between the two counter-rotating areas, a strong induced flow, resembling a jet, is visible. This flow gives strong momentum in the $x$-direction. In the next stage for $k=44$, although the hand is out of the plane for evaluation, counterclockwise rotation still exists near the dorsal side of the hand and clockwise rotation breaks up in several positions. These produce momentum in the $x$-direction.

We calculate the momentum variation $P(t)$ leading to the propulsion. Equation (1) is useful to estimate the flow force acting on the hand. In that equation, the momentum $P$ is calculated directly from

$$
P(t)=\sum_{i=1}^{N} \rho u_{i} \Delta A,
$$

where $N$ represents the total mesh points in the measurement plane and $u_{i}$ is the velocity component in the streamwise direction at the $i$-th mesh point. The sectional area of a unit mesh denoted as $\triangle A$ is equal to $1 \mathrm{~cm}^{2}$. Here it is noteworthy that the momentum $P$ is not exactly the general momentum but that in the meaning of two-dimensional analysis. Therefore, the unit is written 


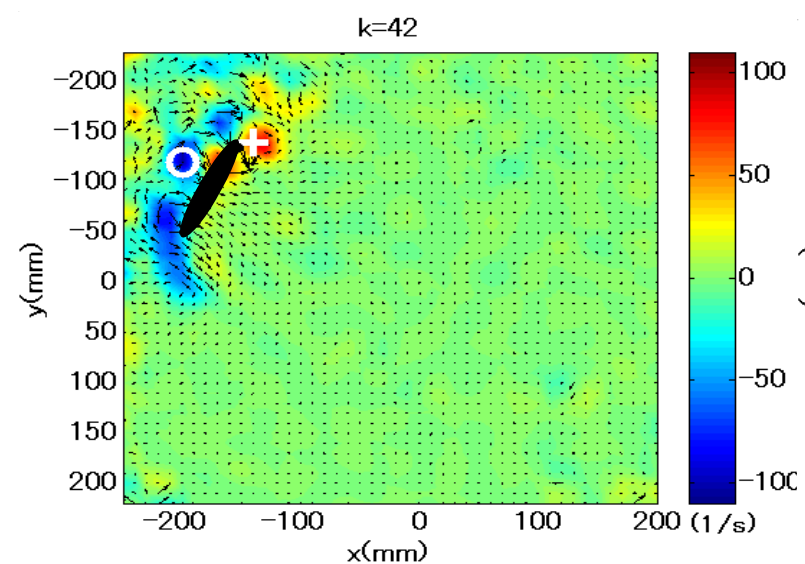

(a)

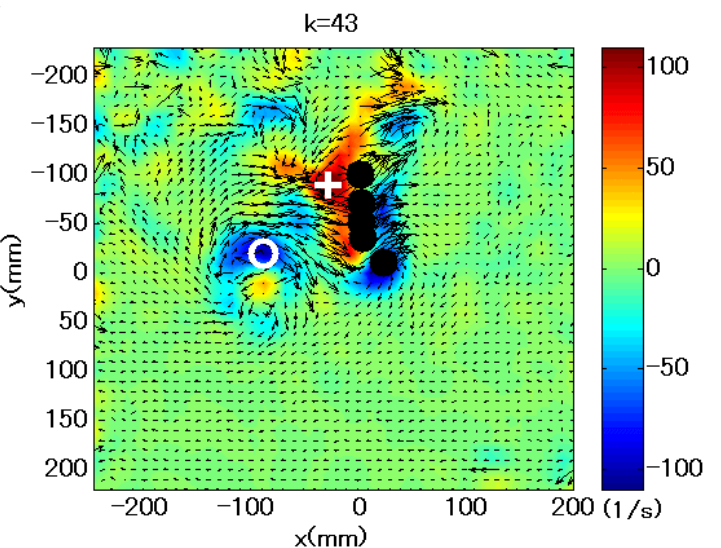

(b)

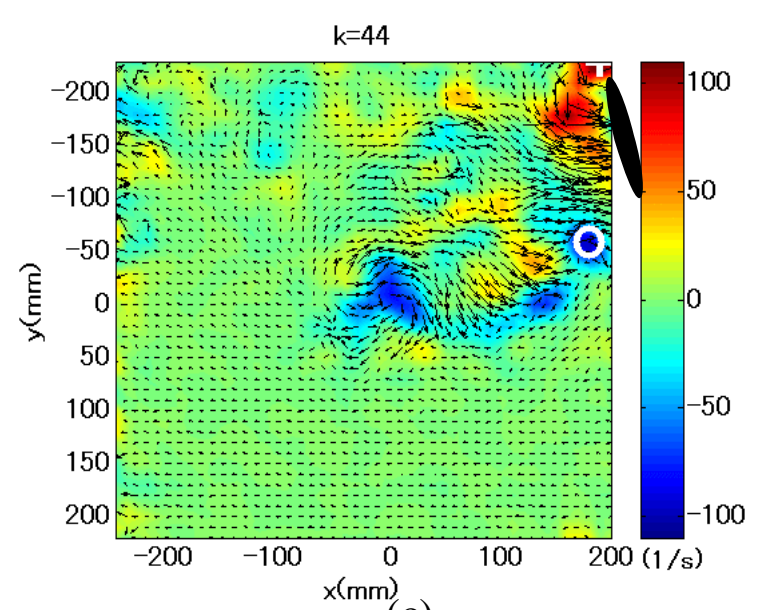

(c)

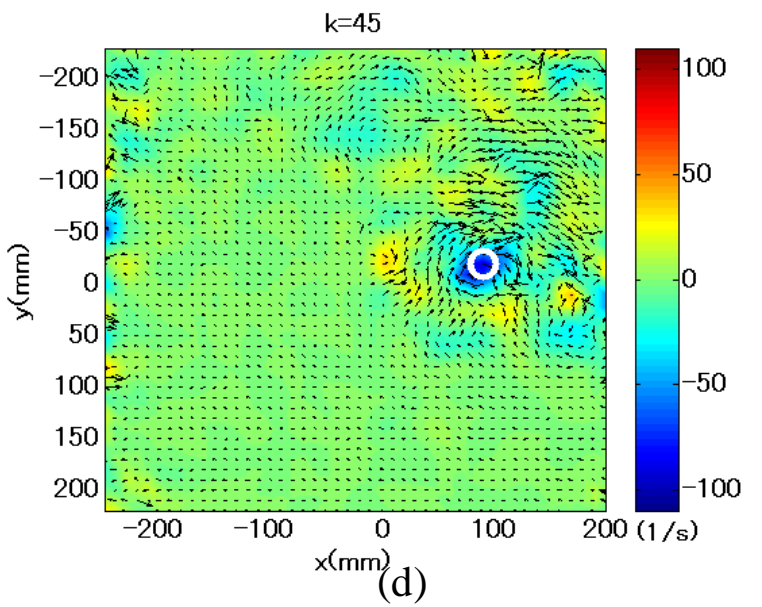

(d)

Fig. 6. Sequential variations of the velocity field at every $70 \mathrm{~ms}$ for subject A. The flume speed was set at $1.2 \mathrm{~m} / \mathrm{s}$. The crosses and circles have identical meaning to those in Fig. 5. In that figure, the strongest vortex pair is visible in panel (b). The maximum and minimum vortices are, respectively, 135.3 and $-108.1 \mathrm{~s}^{-1}$. as $\mathrm{kg} \cdot \mathrm{m} / \mathrm{s}(1 / \mathrm{m})$. The 'momentum' variation is shown in Fig. 7. It increases rapidly at stage 42 to that at stage 43 . During this period, the hand reacts with the strongest force. It is noteworthy that, at stage 43 , the magnitude of vortices becomes strongest corresponding to the rapid increase of momentum. This instance is exactly in the center period of a transition phase from in-sweep to out-sweep. The momentum has a peak at stage 44; it then decreases because the hand, as a momentum generator, is out of the measurement plane. Both sides of the peak at $k=44$ are other peaks corresponding to the transition phase of another hand.

Other examples for subject B in two successive periods are depicted in Fig. 8(a) and Fig. 8(b). Subject B is a male triathlete. The hand movement is also in the transition phase in which an counterclockwise vortex exists near the little finger and a vortex rotating clockwise has just been shed. 


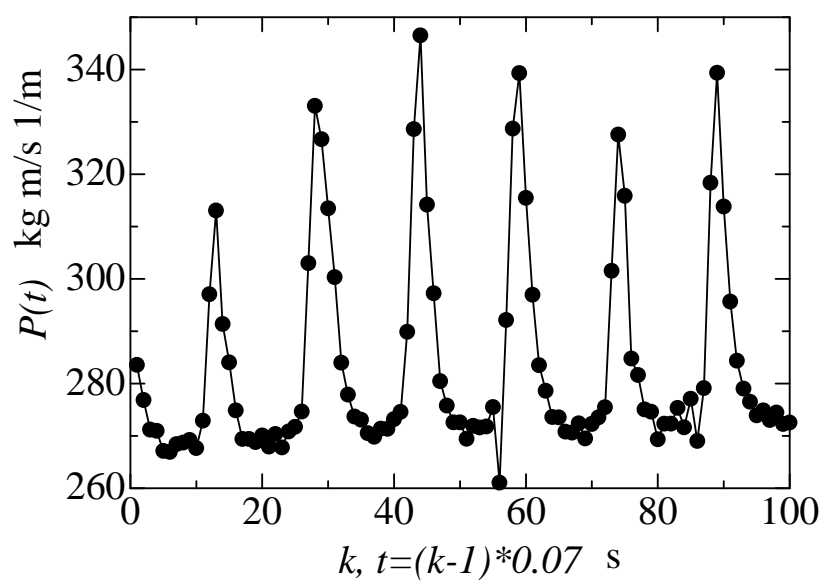

Fig. 7. Time variation of momentum $P(t)$ for subject A. This event is the same as that portrayed in Fig. 6. The number of mesh points $N$ is $46 \times 46=2116$.
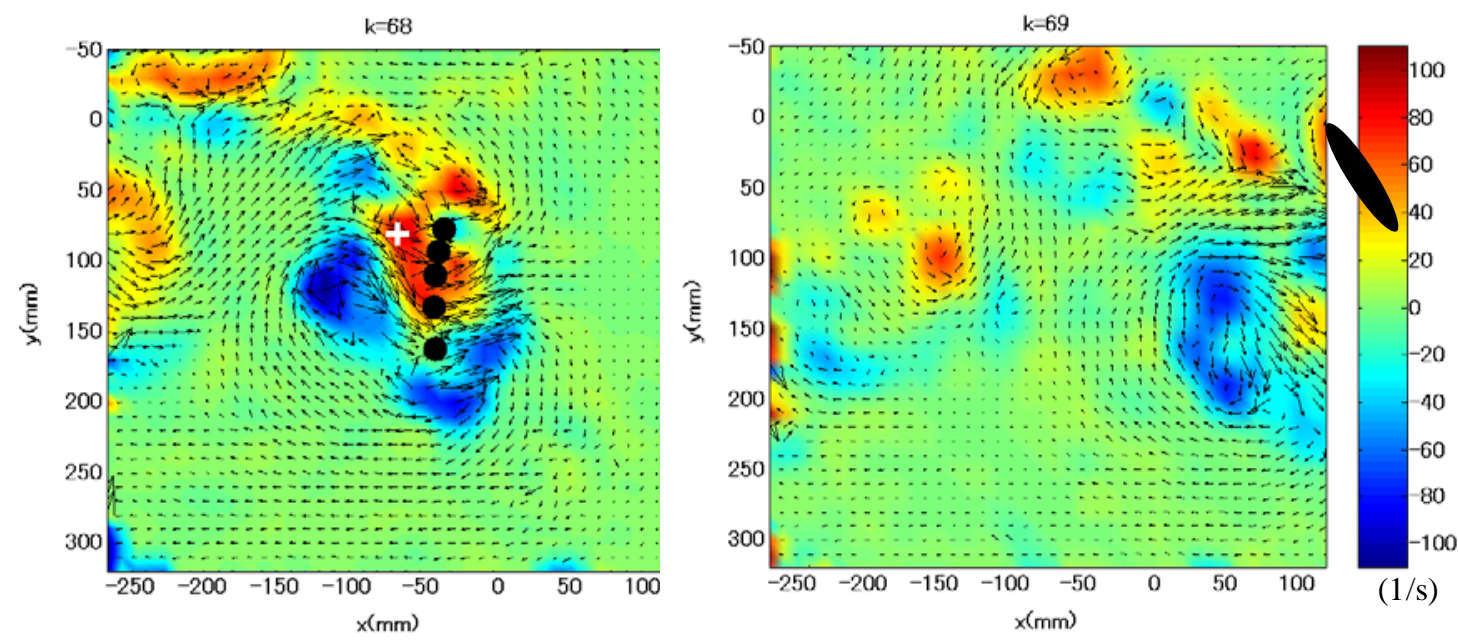

Fig. 8. Flow patterns of two subsequent instants for subject B. The color bar in the right column denotes the magnitude of vorticity in $1 / \mathrm{s}$. The flume speed is $1.25 \mathrm{~m} / \mathrm{s}$. The maximum magnitude of vorticity is $92.7^{-1}$ and the position is near the little finger just behind the palm. A strong vortex with a negative sign exists at the lower left of the positive vortex. This pair produces strong momentum with downward direction.

\section{Discussion}

The importance of unsteady phenomena has been pointed out even in swimming research by many authors (Ungerechts (1986), Arellano (1999), Arellano et al. (2002), and Toussaint et al. (2002)). However, those are related not to quantitative estimation of force but to qualitative discussions on the generation of force. 
Behavior of a certain kind of vortices generated by unsteady movement of blades plays an essential role in receiving high lift force. It is presumable that swimmers also receive the benefits of unsteady lift force. The lift coefficient is preferable to estimate the force $L$ quantitatively. Because the flow field was measured in a horizontal plane, the coefficient $c_{L}$ is defined as

$$
c_{L}=\frac{L}{\frac{1}{2} \rho u^{2} c}
$$

where $u$ is the flow velocity and $c$ a characteristic length. Here, it is noteworthy that the force $L$ has a unit $\mathrm{N} / \mathrm{m}$ in the present $2 \mathrm{D}$ analysis. The coefficient of usual two-dimensional hydrofoils, where $c$ stands for the chord length, is about 1.3 in steady flow (see, for example, Prandtl and Tietjens (1957)). According to Schleihauf et al. (1983), the corresponding value for a hand is about 1.0. On the other hand, for unsteady flow, Izumi and Kuwahara (1983) obtained the values larger than 2.5. This is twice as large as that of a hydrofoil in steady flow.

For $k=42$ and 44 , the momentum per unit depth is given as $P(42)=290$ $\mathrm{kg} \cdot \mathrm{m} / \mathrm{s}(1 / \mathrm{m})$ and $P(44)=347 \mathrm{~kg} \cdot \mathrm{m} / \mathrm{s}(1 / \mathrm{m})$ (see Fig.7). Consequently, from the momentum increase for the interval $2 \times \Delta t(=2 \mathrm{~ms})$ the force $L$ in Eq. (5) is calculated as $407 \mathrm{~N} / \mathrm{m}$. As typical values, setting $c=11 \mathrm{~cm}$ as the square root of the projected area of the hand, and $u=1.7 \mathrm{~m} / \mathrm{s}$ as the magnitude of flow velocity relative to the hand, we can obtain the coefficient 2.6 for the momentum increase between $k=42$ and 44 in the case portrayed in Fig. 7 . This value $\left(c_{L}=2.6\right)$ is very high compared with that predicted by the steady hydrofoil theory. In the estimation based on the momentum change, the drag force was also included. We cannot divide it into lift and drag forces.

The evaluation of propulsive force in only one horizontal plane will be extended to that of force acting on the whole hand if we were able to get the images simultaneously in many horizontal planes using for horizontal $N$ images,

$$
c_{L}=\sum_{i=1}^{N} \frac{L_{i} h}{\frac{1}{2} \rho u^{2} A},
$$

where $h$ is the horizontal depth between two neighboring planes, $A$ the hand projection area, and $L_{i}$ the force per unit depth in $i$-th plane. 


\section{Concluding Remarks}

The force acting on hands has long been investigated. Discussions of the generation have been based on quasi-steady analyses to date (see Schleihauf et al. (1983), Berger et al. (1999)). However, flow visualization using PIV technique revealed that a considerable amount of change is produced by directional changes caused by hand actions and therefore the momentum is generated in the transition phase. In other words, the force acts on hands strongly in the phase.

The phase was crucial for determining the origin of unsteady flow forces because the directional change of the hand makes the flow unsteady. During the transition, a pair of counter-rotating vortices plays an important role, like that of a starting vortex (Dickinson (1996).

For high-speed swimming, swimmers should use unsteady flow force based on the generation and shedding of vortices, in addition to the use of ordinal propulsive forces. Such unsteady force generation occurs in transition phases when using a hand action that incoporates direction changes. Swimmers and their coaches devote much attention to the phases. The present research is a first step to the useful application of unsteady flow forces.

\section{Acknowledgements}

The authors thank Professor H. Toussaint of Vrije Universiteit, Amsterdam and also Professor R. Arellano of the University of Granada for discussions and valuable information that they offered on this subject. This study was supported by a Grant-in-Aid for Scientific Research ((B)(2)15300216)) from the Japan Society for the Promotion of Science.

\section{References}

Arellano, R., 1999. Vortices and propulsion. Applied Proceedings of the XVII International Symposium on Biomechanics in Sports, 53-66.

Arellano, R., Pardillo, S., Gavilan, S., 2002. Underwater undulatory swimming: kinematic characteristics, vortex generation and application during the start, turn and swimming strokes. Proceedings of the XXth International Symposium on Biomechanics in Sports, Universidad de Granada.

Berger, M. A. M., de Groot, G., Hollander, A. P., 1995. Hydrodynamic drag and lift forces on human hand/arm models. Journal of Biomechanics 28, $125-133$.

Berger, M. A. M., Hollander, A. P., de Groot, G., 1999. Determining propulsive 
force in front crawl swimming, A comparison of two methods. Journal of Sports Sciences 17, 97-105.

Bixler, B., Riewald, S., 2002. Analysis of a swimmer's hand and arm in steady flow conditions using computational fluid dynamics. Journal of Biomechanics 35, 713-717.

Counsilman, J. E., 1971. The application of Bernoulli's principle to human propulsion in water. First International Symposium on Biomechanics of Swimming, Universitete Libre de Bruxelles, Brussels, 59-71.

Dickinson, M. H. and Götz, K. G., 1993. Unsteady aerodynamic performance of model wings at low Reynolds numbers. Journal of Experimental Biology 174, 45-64.

Dickinson, M. H., 1996. Unsteady mechanism of force generation in aquatic and aerial locomotion. American Zoology 36, 537-554.

Ellington, C. P., 1984. The aerodynamics of hovering insect flight. IV. Aerodynamic mechanisms. Philosophical Transactions of the Royal Society of London, B305, 79-113.

Izumi, K., Kuwahara, K., 1983. Unsteady flow field, lift and drag measurements of impulsively started elliptic cylinder and circular-arc airfoil. AIAA83-1711, 1-15.

Lamb H., 1932. Hydrodynamics. 6th Ed., Art. 157, Cambridge University Press.

Lugt H. J., 1995. Vortex Flow in Nature and Technology, Krieger Publishing Company, 247.

Prandtl, L. and Tietjens, O.G., 1957. Applied Hydro- and Aeromechanics, Sec. 90, Dover Publications.

Sakakibara, J., Nakagawa, M., Yoshida, M., 2004. Stereo-PIV study of flow around a maneuvering fish. Experiments in Fluids 36, 282-293.

Sanders, R. H., 1999. Hydrodynamic characteristics of a swimmer's hand. Journal of Applied Biomechanics 15, 3-26.

Schleihauf, R. E., 1979. A hydrodynamic analysis of swimming propulsion. In: Terauds, J., Bedingfield, E. W. (Eds.), Swimming III. University Park Press, Baltimore, pp. 70-109.

Schleihauf, R. E., Gray, L., DeRose, J., 1983. Three dimensional analysis of swimming propulsion in the sprint front crawl stroke. In: Hollander, P., Huijing, P., De Groot, G. (Eds.), Biomechanics and Medicine in Swimming IV, pp. 173-183.

Toussaint, H. M., Van den Berg, C., Beek, W. J., 2002. "Pumped-up propulsion" during front crawl swimming. Medicine and Science in Sports and Exercise 34, 314-319.

Tritton, D. J., 1988. Physical Fluid Dynamics, 2nd Ed. Oxford University Press, pp. 81-82.

Ungerechts, B. E., 1986. The relation of peak body acceleration to phases of movements in swimming. In: Ungerechts, B.E. (Eds.) Swimming Science V. Human Kinetics Books, Champaign, pp. 61-66.

Wagner, H., 1925, Über die Entstehung des dynamischen Auftriebes von 
Tragflügeln. Zeitschrift für Angewante Mathematik und Mechanik 5, 17-35. 